\title{
État des lieux de l'étiquetage des médicaments dangereux sur le marché canadien
}

\author{
Alexia Janes, Cécile Bérard et Jean-François Bussières
}

\section{INTRODUCTION}

A u Canada, l'étiquetage des médicaments est régi par la Loi sur les aliments et drogues ${ }^{1}$, le Règlement sur les aliments et drogues ${ }^{2}$ et d'autres lignes directrices ${ }^{3,4}$. Ces lignes directrices s'inscrivent dans la foulée des changements législatifs apportés par le projet de loi C-17, Loi visant à protéger les Canadiens contre les drogues dangereuses (Loi de Vanessa) $)^{5}$. Ce projet de loi vise notamment à améliorer le suivi effectué par Santé Canada de l'innocuité d'un produit après sa mise sur le marché et permet d'exiger du fabricant la modification de l'étiquetage d'un produit de santé déjà sur le marché. De plus, la nouvelle ligne directrice de 2014 relative à l'étiquetage des médicaments pharmaceutiques destinés à l'usage des humains expose les exigences minimales d'étiquetage des produits pharmaceutiques, mais précise qu' " il existe de nombreuses lignes directrices publiées par des tiers, dont plusieurs par des associations professionnelles indépendantes ou des groupes d'intérêt public, qui fournissent des directives supplémentaires facultatives en matière d'étiquetage. Les mesures d'étiquetage supplémentaires [...] sont acceptables, pourvu qu'elles n'entrent pas en conflit avec les exigences réglementaires $\|^{3}$. Cependant, cette nouvelle ligne directrice précise que "Santé Canada ne donne aucunement son aval à ces documents et n'approuve pas nécessairement leur contenu $~^{3}$.

Malgré ce cadre législatif entourant les médicaments, on note un vide juridique concernant l'étiquetage des médicaments dangereux. Les médicaments dangereux sont définis par l'American Society of Hospital Pharmacist et par le National Institute for Occupational Safety and Health (NIOSH), comme présentant une ou plusieurs des caractéristiques suivantes : cancérogène, génotoxique, tératogène, toxique pour la reproduction, toxique à faible dose pour un organe, ou ayant une structure similaire à un autre médicament dangereux ${ }^{6,7}$. En septembre 2004, le NIOSH a publié une alerte visant la protection des travailleurs exposés aux médicaments dangereux. Dans cette alerte, les médicaments dangereux étaient définis comme des médicaments susceptibles d'entrainer l'apparition d'effets indésirables chez des travailleurs y étant exposés, notamment par l'intermédiaire du contact avec des surfaces contaminées. Cette alerte préconisait alors un étiquetage permettant l'identification de ces médicaments afin de promouvoir l'utilisation de moyens de protection adéquats lors de leur manipulation et de limiter ainsi l'exposition des travailleurs ${ }^{8}$. Le NIOSH publie aussi périodiquement une liste de médicaments dangereux; la dernière mise à jour de 2014 comptait 184 médicaments, dont 97 antinéoplasiques ${ }^{7}$. Si cette liste comporte sans surprise des médicaments antinéoplasiques, plusieurs s'étonnent d'y retrouver des immunosuppresseurs, des antipsychotiques, des antidépresseurs, des hormones, etc. L'alerte NIOSH de 2004 a entrainé de nombreuses sociétés savantes, notamment américaines, européennes et canadiennes, à revoir leurs politiques et procédures dans le but de promouvoir l'utilisation d'équipements de protection lors de la manipulation des produits ${ }^{9-19}$. Ces lignes directrices préconisent notamment d'apposer un logo cytotoxique, une mention PRÉCAUTION ou encore un logo de couleur vive accompagné d'un court texte informatif sur les conditionnements des médicaments dangereux ${ }^{18,19}$ afin de faciliter leur identification. Il est donc étonnant de constater que dix ans après la publication de l'alerte NIOSH, l'étiquetage commercial des médicaments dangereux semble peu conforme à ces recommandations. Nous nous sommes donc intéressés à l'étiquetage des médicaments dangereux au Canada.

\section{MÉTHODE}

Il s'agit d'une étude descriptive transversale et observationnelle. Cette étude vise principalement à effectuer un état des lieux de l'étiquetage commercial des médicaments dangereux chez un grossiste canadien.

Des étiquettes internes et externes de médicaments dangereux ont été observées chez un grossiste canadien 
(McKesson Canada, entrepôt Pie-IX, Montréal [Québec]) à un jour donné (21 août 2014). Les médicaments dangereux ont été identifiés à partir de la liste de médicaments dangereux publiée par le NIOSH en $2012^{20}$, la mise à jour de 2014 n'ayant pas encore été publiée lors de la mise en œuvre de l'étude. La liste des médicaments dangereux disponibles dans le catalogue du grossiste a été confrontée à la liste du NIOSH pour l'établissement de la liste définitive des produits à observer.

Les définitions du Règlement sur les aliments et drogues ont été utilisées pour caractériser les étiquettes évaluées ${ }^{2}$. Une étiquette interne a été défini comme l'étiquette apposée sur le contenant même du produit pharmaceutique. Une étiquette externe a été définie comme l'étiquette apposée sur l'extérieur de l'emballage du produit pharmaceutique. Il pouvait y avoir plus d'une étiquette externe dans un système d'emballage multiple (p. ex. une fiole emballée dans un sachet, lui-même emballé dans une ou plusieurs boîtes placées dans une plus grande). Nous avons défini comme suremballage une étiquette externe de second ordre dans le cas d'un système d'emballage multiple.

Une grille de collecte des données a été établie à partir de l'ensemble des documents de référence suivants : le Guide de prévention sur la manipulation sécuritaire des médicaments dangereux publié par l'Association paritaire pour la santé et la sécurité du travail du secteur affaires sociales (ASSTSAS) en $2008^{19}$, un bulletin d'informations professionnelles portant sur la manipulation des médicaments dangereux émis par de l'Ordre des pharmaciens du Québec ${ }^{16}$, les recommandations issues du NIOSH de $2004^{8}$ et leurs mises à jour de $2014^{7}$.

Pour chaque étiquette observée, nous avons relevé la dénomination commune et commerciale complète, la concentration ou la teneur, la forme, le format et le fabricant à l'aide de la grille de collecte. En outre, nous avons relevé la présence ou l'absence de logos ou de mentions pertinentes relatives à la protection des travailleurs.

Nous avons limité notre observation aux produits physiquement présents dans le stock du grossiste le jour de la collecte. En raison du temps d'observation restreint, nous avons convenu de cesser l'observation des produits présentant la même dénomination commune internationale (DCI) lorsque plus de cinq produits de fabricants différents (tout dosage et toute voie d'administration confondus) avaient été analysés. Un seul produit a fait exception à la règle, le finasteride, molécule dont la plupart des étiquettes portaient une mention liée à la manipulation. De plus, parmi les substances portant la même DCI, cette molécule nous permettait l'accès au plus grand nombre de fabricants différents. L'observation de l'ensemble des étiquettes propres au finasteride nous a permis une analyse plus exhaustive de l'étiquetage d'un médicament dangereux et une comparaison des différents fabricants.

Après avoir recueilli l'ensemble des données, nous avons établi localement des critères de conformité d'étiquetage des médicaments dangereux, basés sur les recommandations d'étiquetage des médicaments dangereux émises par les sociétés savantes $^{18,19}$. Nous avons défini comme étant conformes, les étiquettes comportant au moins un des trois critères suivants : logo cytotoxique, mention «cytotoxique/toxique " ou mention de précautions relatives à la manipulation. Nous avons ensuite calculé la proportion d'étiquettes conformes de tous les médicaments dangereux évalués, puis plus spécifiquement des médicaments antinéoplasiques et non antinéoplasiques. Nous avons également calculé le taux de conformité des étiquettes en fonction de la voie d'administration des médicaments. Ensuite, nous avons comparé la conformité de l'étiquetage des médicaments génériques à celle des médicaments novateurs. Enfin, nous avons évalué le taux de conformité des étiquettes par fabricant de qui nous avons observé au moins dix produits.

Les taux de conformité ont été calculés. Cette étude a abouti à la réalisation de statistiques uniquement descriptives.

\section{RÉSULTATS}

Le 21 août 2014, 335 médicaments provenant de 69 fabricants différents ont été observés sur les 581 (c.-à-d. $58 \%$ du total) médicaments dangereux présents dans le catalogue du grossiste.

Sur ces 335 médicaments, 383 étiquettes ont été analysées (18 suremballages, 240 étiquettes externes et 125 étiquettes internes). Les 335 médicaments comportaient 106 DCI différentes. Pour 28 \% (30/106) des DCI analysés, nous avons restreint notre observation à cinq produits de fabricants différents.

Sur la totalité des étiquettes analysées, $80 \%$ (305/383) ont été jugées non conformes (tableau 1). Parmi les étiquettes conformes, $72 \%(56 / 78)$ correspondaient à des agents antinéoplasiques. La majorité des étiquettes de médicaments non antinéoplasiques était non conforme. Le taux de conformité des étiquettes des médicaments injectables était supérieur à celui des médicaments administrés par d'autres voies. Enfin, le taux de conformité des étiquettes des médicaments génériques était supérieur à celui des médicaments novateurs étudiés.

Lorsque nous réduisons notre champ d'étude aux fabricants d'un nombre minimum de dix produits observés, nous constatons que certains d'entre eux ont présenté des taux de conformité d'étiquetage de médicaments dangereux supérieurs aux autres (tableau 2). Sur les neuf fabricants concernés, un seul présentait un taux de conformité des étiquettes observées supérieur à $50 \%$. Cinq fabricants présentaient des taux de non-conformité des étiquettes observées supérieurs à $90 \%$.

Sur l'ensemble des étiquettes observées, seulement $9 \%$ (33/383) des étiquettes portaient une mention évoquant des précautions particulières de manipulation (soit deux suremballages, 26 étiquettes externes et 5 étiquettes internes). Ces 33 étiquettes concernaient 23 fabricants. Les mentions évoquant des précautions particulières de manipulation étaient diverses et variaient d'un fabricant à l'autre (tableau 3). 
This single copy is for your personal, non-commercial use only.

For permission to reprint multiple copies or to order presentation-ready copies for distribution, contact CJHP at cjhpedit@cshp.ca

Tableau 1. Profil détaillé de la conformité de l'étiquetage des médicaments dangereux observés

\begin{tabular}{|c|c|c|c|c|}
\hline \multirow[b]{2}{*}{$\begin{array}{l}\text { Comparaison et type } \\
\text { de médicament }\end{array}$} & \multicolumn{4}{|c|}{ Conformité des étiquettes, $n / N(\%)$} \\
\hline & Suremballage & Externe & Interne & Total \\
\hline \multicolumn{5}{|l|}{ Antinéoplasiques ou non } \\
\hline Antinéoplasiques & $7 / 12(58)$ & $38 / 125(30)$ & $11 / 37(30)$ & $56 / 174(32)$ \\
\hline Non antinéoplasiques & $0 / 6 \quad(0)$ & $17 / 115(15)$ & $5 / 88 \quad(6)$ & $22 / 209(11)$ \\
\hline \multicolumn{5}{|l|}{ Génériques ou novateurs } \\
\hline Génériques & $4 / 9(44)$ & $39 / 112(35)$ & 16/91 (18) & $59 / 212(28)$ \\
\hline Novateurs & 3/9 (33) & $16 / 128(13)$ & $0 / 34 \quad(0)$ & 19/171 (11) \\
\hline \multicolumn{5}{|l|}{ Voie d'administration } \\
\hline Voie parentérale & $5 / 8(63)$ & 39/94 (41) & $10 / 20(50)$ & $54 / 122(44)$ \\
\hline Voie orale & $1 / 7(14)$ & $14 / 118(12)$ & $6 / 103 \quad(6)$ & $21 / 228 \quad(9)$ \\
\hline Autres voies & 1/3 (33) & $2 / 28 \quad(7)$ & $0 / 2 \quad(0)$ & $3 / 33 \quad(9)$ \\
\hline Total & $7 / 18(39)$ & $55 / 240(23)$ & $16 / 125(13)$ & $78 / 383(20)$ \\
\hline
\end{tabular}

\begin{tabular}{|c|c|c|c|}
\hline Fabricant & $\begin{array}{c}\text { Nombre de } \\
\text { produits observés }\end{array}$ & $\begin{array}{c}\text { Nombre total } \\
\text { d'étiquettes } \\
\text { observées }\end{array}$ & $\begin{array}{c}\text { Nombre (\%) } \\
\text { d'etiquettes } \\
\text { conforme }\end{array}$ \\
\hline Apotex & 27 & 27 & $1 \quad(4)$ \\
\hline Hospira & 20 & 25 & (4) \\
\hline Merck & 10 & 11 & $2(18)$ \\
\hline Novartis & 14 & 18 & 1 (6) \\
\hline Novopharm & 16 & 19 & $13(68)$ \\
\hline Pfizer & 22 & 25 & 7 (28) \\
\hline Pharmascience & 12 & 15 & $1 \quad(7)$ \\
\hline Sandoz & 19 & 23 & 9 (39) \\
\hline Teva & 20 & 25 & $2 \quad(8)$ \\
\hline
\end{tabular}

Quant au finasteride, 14 produits de 12 fabricants différents ont été observés. Parmi les 15 étiquettes de ces produits, 12 (80\%) ont été jugées conformes. Elles contenaient toutes la mention « les femmes enceintes ou susceptibles de l'être ne doivent pas manipuler les comprimés brisés ou écrasés ». Seul un fabricant ne présentait aucune mention de précautions particulières de manipulation sur les étiquettes de l'échantillon observé.

\section{DISCUSSION}

À notre connaissance, il s'agit de la première étude évaluant l'étiquetage des médicaments dangereux au Canada. Notre étude met en évidence le fait que la majorité $(80 \%)$ des étiquettes internes ou externes de médicaments dangereux observés ne comportait aucune mention permettant de les reconnaitre de manière claire et immédiate.

\section{Conformité de l'étiquetage des médicaments antinéoplasiques comparativement aux non antinéoplasiques}

La majorité des médicaments dont les étiquettes avaient été jugées conformes appartenait aux agents antinéoplasiques utilisés en oncologie. Or le secteur de l'oncologie est un secteur particulier, généralement séparé des autres secteurs, où travaille un personnel restreint, davantage formé et sensibilisé à la manipulation d'agents cytotoxiques et à l'utilisation d'équipements de protection. En revanche les médicaments dangereux non antinéoplasiques, au même titre que certains médicaments antinéoplasiques utilisés dans d'autres secteurs (p. ex. en immunologie, en rhumatologie) peuvent être manipulés par l'ensemble du personnel de la pharmacie et des différents services de soins. Il est primordial que tous les médicaments dangereux puissent être reconnus par l'ensemble du personnel susceptible de les manipuler, afin qu'ils puissent être séparés des autres médicaments et manipulés de manière sécuritaire selon des procédures établies. Il est donc essentiel que les fabricants fassent un effort pour favoriser l'étiquetage approprié et sécuritaire des médicaments dangereux de toutes les classes de médicaments destinés à tous les usages.

\section{Conformité de l'étiquetage en fonction de la voie d'administration}

Nous avons observé un taux de conformité de l'étiquetage des médicaments injectables supérieur à celui des médicaments 
This single copy is for your personal, non-commercial use only.

For permission to reprint multiple copies or to order presentation-ready copies for distribution, contact CJHP at cjhpedit@cshp.ca

\section{Tableau 3. Exemples de mentions des précautions pour la manipulation des médicaments dangereux observés}

\begin{tabular}{|c|c|}
\hline Fabricant & Exemples de mentions des précautions particulières lors de manipulations \\
\hline Accord Healthcare & « Manipuler avec prudence » \\
\hline GlaxoSmithKline & « Manipulation : User de prudence » \\
\hline Taro Pharmaceuticals & $\begin{array}{l}\text { "Instructions de manipulation particulières. Pour obtenir des renseignements } \\
\text { posologiques et des directives de manipulation détaillées veuillez consulter } \\
\text { le feuillet d'information ci-joint. » }\end{array}$ \\
\hline Novartis & $\begin{array}{l}\text { "Special Handling Instructions. For detailed handling instructions and } \\
\text { directions, please consult the enclosed Package Insert. »* }\end{array}$ \\
\hline Lundbeck & $\begin{array}{l}\text { "Agent cytotoxique - Manipuler et préparer le produit avec prudence. II est } \\
\text { recommandé de porter des gants et des lunettes de protection afin d'éviter } \\
\text { l'exposition au produit. » }\end{array}$ \\
\hline Novopharm & « Handle with EXTRA CARE, glove BEFORE OPENING » \\
\hline Sandoz & $\begin{array}{l}\text { "Les femmes en âge de procréer ne doivent pas toucher ce médicament } \\
\text { avec les mains nues. Utiliser des gants. » }\end{array}$ \\
\hline Baxter & $\begin{array}{l}\text { "Posologie, manipulation : Consulter les renseignements posologiques } \\
\text { ci-inclus pour obtenir des renseignements complets, notamment sur la } \\
\text { posologie et la manipulation. » }\end{array}$ \\
\hline
\end{tabular}

destinés aux autres voies d'administration. Or pour le maintien d'un environnement stérile, la manipulation des médicaments injectables nécessite couramment l'utilisation d'équipements de protection stériles. Les conditions de stérilité n'étant pas requises pour les médicaments destinés aux autres voies, on utilise rarement des équipements de protection stériles pour leur manipulation, d'où la nécessité pour les fabricants de renforcer l'identification des produits.

\section{Conformité d'étiquetage des médicaments génériques comparativement aux médicaments novateurs}

Nous avons finalement constaté que certains fabricants semblaient se conformer davantage aux recommandations portant sur l'identification des médicaments dangereux par rapport à d'autres. Parmi les fabricants dont le taux de conformité de l'étiquetage des produits testés est supérieur, on retrouvait davantage de fabricants génériques. Cela s'explique peut-être par une plus grande motivation des fabricants de génériques à mieux se positionner par rapport aux novateurs, de manière à pouvoir s'emparer du marché lors du retour d'appel d'offre. Une autre explication probable porte sur le fait que l'étiquetage des médicaments génériques semble plus uniformisé, ce qui entraine une plus grande proportion d'étiquettes conformes.

\section{Étiquetage des médicaments dangereux par les pharmacies}

Cette variabilité de la conformité de l'étiquetage des médicaments dangereux en fonction du type de médicaments ou du type de voie d'administration peut notamment s'expliquer par le fait qu'aucune loi ou qu'aucun règlement opposable au Canada n'exige des fabricants un étiquetage spécifique des médicaments dangereux et que le statut même de médicament dangereux n'existe pas encore au Canada. Ce vide juridique persiste actuellement malgré l'existence avérée de contamination de fioles au départ des usines ${ }^{21-24}$.

Il constitue également un paradoxe par rapport à la règlementation imposée aux pharmaciens pour les préparations magistrales de médicaments dangereux avec la publication récente de la norme sur la préparation de produits stériles dangereux en pharmacie ${ }^{25}$. Cette norme précise que toutes les préparations de produits stériles dangereux doivent porter la mention "Cytotoxique ". Ce cadre normatif s'accompagne de nombreuses politiques et procédures préconisant un étiquetage sécuritaire des médicaments dangereux préparés et dispensés par les pharmacies. Au Québec, l'Ordre des pharmaciens du Québec $^{16}$ demande aux pharmaciens de se référer au Guide de prévention de l'ASSTSAS de $2008^{19}$ et préconise, au sein des pharmacies, l'apposition du symbole $\mathrm{C}$ majuscule accompagné de la légende "CYTOTOXIQUE » sur l'ensemble des produits cytotoxiques et d'une étiquette « PRÉCAUTION » sur les autres médicaments dangereux non cytotoxiques. En ColombieBritannique, la British Columbia Cancer Agency spécifie que les médicaments dangereux préparés par les pharmacies doivent comporter une étiquette mentionnant le médicament comme étant dangereux ${ }^{17,26}$. Enfin, au niveau national, la Société canadienne des pharmaciens d'hôpitaux indique aux pharmaciens que les médicaments dangereux dispensés (y compris les médicaments cytotoxiques) devaient comporter un étiquetage approprié qui mentionne clairement leur caractère dangereux ${ }^{15}$.

Parallèlement à l'ensemble de ces recommandations, il existe actuellement une démarche d'uniformisation internationale de l'étiquetage des produits dangereux (qui ne sont pas des médicaments) par la mise en place d'un système unique, le SGH 
(Système général harmonisé). Le SGH stipule que certains renseignements, tels que des mentions de danger et d'avertissement, des symboles uniformisés ou encore des suggestions de mises en garde doivent apparaitre sur l'étiquette des produits dangereux ${ }^{27}$. Le gouvernement du Canada a intégré le SGH au Système d'information sur les matières dangereuses utilisées au travail (SIMDUT) le 11 février 201528. Par conséquent il est surprenant de constater que la mise en place d'un tel projet par le gouvernement canadien n'inclut pas l'étiquetage des médicaments dangereux.

\section{Forces et limites}

À notre connaissance il s'agit de la première étude offrant une évaluation de la conformité de l'étiquetage de médicaments dangereux commercialisés au Canada. Il s'agit d'un échantillon relativement représentatif de l'ensemble des produits actuellement sur le marché (plus de $50 \%$ des produits commercialisés par le grossiste canadien examiné).

Néanmoins, cette évaluation n'incluait pas l'ensemble des médicaments dangereux actuellement disponibles sur le marché canadien. De plus, nous avons restreint notre observation à cinq médicaments de même DCI de fabricants différents, ce qui a pu introduire un biais de sélection des données. Enfin, les critères de conformité d'étiquetage ont été définis localement, après la collecte de l'ensemble des données.

\section{CONCLUSION}

Moins du quart des étiquettes de médicaments dangereux évaluées au Canada ont été jugées conformes quant à la mention $\mathrm{du}$ risque d'exposition professionnelle. Cette absence de conformité s'explique notamment par l'absence actuelle d'obligations règlementaires destinée aux fabricants, malgré l'existence de politiques et de procédures encadrant l'étiquetage des médicaments dangereux par les pharmacies. Il semble donc important de statuer sur une règlementation universelle concernant l'étiquetage des médicaments dangereux au Canada afin de promouvoir l'utilisation de moyens de protection adéquats lors des manipulations et de limiter ainsi l'exposition professionnelle des travailleurs aux médicaments dangereux.

\section{Références}

1. Loi sur les aliments et drogues (L.R.C. (1985), ch. F-27). Publié au : http://laws-lois.justice.gc.ca/fra/lois/f-27/. Consulté le 26 juin 2015.

2. Règlement sur les aliments et drogues (C.R.C., ch. 870). Publié au : http://laws-lois.justice.gc.ca/fra/reglements/C.R.C.,_ch._870/. Consulté le 26 juin 2015.

3. Ligne directrice - Étiquetage des médicaments pharmaceutiques destinés à l'usage des humains. Ottawa (ON) : Santé Canada; 2014. Publié au : www.hc-sc.gc.ca/dhp-mps/prodpharma/applic-demande/guideld/label_guide_ld-fra.php. Consulté le 26 juin 2015.

4. Ligne directrice - Norme d'étiquetage des médicaments pour adultes vendus sans ordonnance contre la toux et le rhume administrés par voie orale. Ottawa (ON) : Santé Canada; 2014. Publié au : www.hc-sc.gc.ca/dhpmps/prodpharma/applic-demande/guide-ld/label-etiquet-pharm/coughtoux-fra.php. Consulté le 26 juin 2015.
5. Loi visant à protéger les Canadiens contre les drogues dangereuses (Loi de Vanessa). Modifications à la Loi sur les aliments et drogues (projet de Loi C-17). Ottawa (ON) : Santé Canada; 2015. Publié au : www.hcsc.gc.ca/dhp-mps/legislation/unsafedrugs-droguesdangereuses-fra.php. Consulté le 26 juin 2015.

6. American Society of Hospital Pharmacists. ASHP technical assistance bulletin on handling cytotoxic and hazardous drugs. Am J Hosp Pharm. 1990;47(5):1033-49.

7. Connor TH, MacKenzie BA, DeBord DG, Trout DB, O'Callaghan JP. NIOSH list of antineoplastic and other hazardous drugs in healthcare settings, 2014. DHHS (NIOSH) Publication 2014-138. Cincinnati (OH) : US Department of Health and Human Services, Centers for Disease Control and Prevention, National Institute for Occupational Safety and Health; 2014.

8. NIOSH alert: Preventing occupational exposures to antineoplastic and other hazardous drugs in health care settings. DHHS (NIOSH) Publication 2004-165. Cincinnati $(\mathrm{OH})$ : US Department of Health and Human Services, Centers for Disease Control and Prevention, National Institute for Occupational Safety and Health; 2004. Publié au : www.cdc. gov/niosh/docs/2004-165/pdfs/2004-165.pdf. Consulté le 26 juin 2015.

9. American Society of Health-System Pharmacists. ASHP guidelines on handling hazardous drugs. Am J Health Syst Pharm. 2006;63(12):1172-93. Publié au : www.ashp.org/doclibrary/bestpractices/prepgdlhazdrugs.aspx. Consulté le 26 juin 2015.

10. Chaffee BW, Armitstead JA, Benjamin BE, Cotugno MC, Forrey RA, Hintzen BL, et al. Guidelines for the safe handling of hazardous drugs: consensus recommendations. Am J Health Syst Pharm. 2010;67(18):1545-6.

11. Society of Hospital Pharmacists of Australia Committee of Specialty Practice in Oncology. SHPA standards of practice for the safe handling of cytotoxic drugs in pharmacy departments. J Pharm Pract Res. 2005;35(1):44-52.

12. Standards of practice for oncology pharmacy in Canada. Version 2. North Vancouver (BC) : Canadian Association of Pharmacy in Oncology; 2009. Publié au : www.capho.org/sites/default/files/page-files/Standardsof PracticeFORWEBV2Dprintable.pdf. Consulté le 26 juin 2015.

13. QuapoS 3: Quality standard for the oncology pharmacy service with commentary. Hamburg (Germany): Institute for Applied Healthcare Sciences for German Society of Oncology Pharmacy; 2003. Publié au : www.esop.li/ downloads/library/Quapos-en-with-commentary.pdf. Consulté le 26 juin 2015.

14. Green E, Johnston M, Trudeau M, Schwartz L, Poirier S, Macartney G, et al. Safe handling of parenteral cytotoxics: recommendations for Ontario. J Oncol Pract. 2009;5(5):245-9.

15. Hazardous pharmaceuticals (including cytotoxic drugs): guidelines for handling and disposal. Ottawa $(\mathrm{ON})$ : Canadian Society of Hospital Pharmacists; 1997.20 p.

16. Ordre des pharmaciens du Québec. La manipulation des médicaments dangereux en pharmacie. Bull Inform Prof. 2010;(169):1-7.

17. Hazardous drug safe handling standards. Politique V-10. Vancouver (BC) : British Columbia Cancer Agency; 1997 [revisé 2000, 2012, 2013, 2014 ]. Publié au : www.bccancer.bc.ca/chemotherapy-protocols-site/Documents/ Policies\%20and\%20Procedures/V_10_SafeHandlingStandards_August201 4.pdf. Consulté le 26 juin 2015.

18. International Society of Oncology Pharmacy Practicioners Standards Committee. ISOPP standards of practice. Safe handling of cytotoxics. $J$ Oncol Pharm Pract. 2007;13 Suppl:1-81.

19. Guide de prévention. Manipulation sécuritaire des médicaments dangereux. Montréal (QC) : Association paritaire pour la santé et la sécurité du travail du secteur des affaires médicales; 2008.

20. NIOSH list of antineoplastic and other hazardous drugs in healthcare settings 2012. DHHS (NIOSH) Publication 2012-150. Cincinnati (OH) : US Department of Health and Human Services, Centers for Disease Control and Prevention, National Institute for Occupational Safety and Health; 2012. Publié au : www.cdc.gov/niosh/docs/2012-150/pdfs/2012-150.pdf. Consulté le 26 juin 2015.

21. Schulz H, Bigelow S, Dobish R, Chambers CR. Antineoplastic agent workplace contamination study: the Alberta Cancer Board Pharmacy perspective. J Oncol Pharm Pract. 2005;11(3):101-9.

22. Favier B, Gilles L, Ardiet C, Latour JF. External contamination of vials containing cytotoxic agents supplied by pharmaceutical manufacturers. $J$ Oncol Pharm Pract. 2003;9(1):15-20. 
23. Connor TH, Sessink PJM, Harrison BR, Pretty JR, Peters BG, Alfaro RM, et al. Surface contamination of chemotherapy drug vials and evaluation of new vial-cleaning techniques: results of three studies. Am J Health Syst Pharm. 2005;62(5):475-84.

24. Schierl R, Herwig A, Pfaller A, Groebmair S, Fischer E. Surface contamination of antineoplastic drug vials: comparison of unprotected and protected vials. Am J Health Syst Pharm. 2010;67(6):428-9.

25. Préparation de produits stériles dangereux en pharmacie. Norme 2014.02. Montréal (QC) : Ordre des pharmaciens du Québec; 2014. Publié au : www.opq.org/cms/Media/1847_38_fr-CA_0_Norme_2014_02.pdf. Consulté le 26 juin 2015.

26. Safe handling of hazardous drugs. Module 1. Vancouver (BC) : BC Cancer Agency; 2015. Publié au : www.bccancer.bc.ca/pharmacy-site/Documents/ Safe\%20Handling/2\%20\%20Module\%201_Safe\%20Handling\%20of\%2 0Hazardous\%20Drugs_May\%202015.pdf. Consulté le 26 juin 2015.

27. Système général harmonisé (SGH). Hamilton $(\mathrm{ON})$ : Centre canadien d'hygiène et de sécurité au travail; 2015. Publié au : www.cchst.com/ oshanswers/chemicals/ghs.html. Consulté le 26 juin 2015.

28. SIMDUT [Système d'information sur les matières dangereuses utilisées au travail] 2015. Ottawa (ON): Santé Canada; 2015. Publié au : www. hc-sc.gc.ca/ewh-semt/occup-travail/whmis-simdut/ghs-sgh/index-fra.php. Consulté le 7 janvier 2016.
Alexia Janes, D. Pharm., est assistante à la recherche au Département de pharmacie et Unité de recherche en pratique pharmaceutique, Centre hospitalier universitaire Sainte-Justine, Montréal (Québec).

Cécile Bérard est assistante à la recherche au Département de pharmacie et Unité de recherche en pratique pharmaceutique, Centre hospitalier universitaire Sainte-Justine, Montréal (Québec). Elle est aussi candidate D. Pharm. au CHU de Toulouse, Toulouse, France.

Jean-François Bussières, B. Pharm., M. Sc., M. B. A., FCSHP, est chef, Département de pharmacie et Unité de recherche en pratique pharmaceutique, Centre hospitalier universitaire Sainte-Justine, et professeur, Faculté de pharmacie, Université de Montréal, Montréal (Québec).

Intérêts concurrents : Aucun déclaré.

Adresse de correspondance :

Jean-François Bussières

Département de pharmacie

Centre hospitalier universitaire Sainte-Justine

3175, chemin de la Côte Sainte-Catherine

Montréal QC H3T 1C5

Courriel : jf.bussieres@ssss.gouv.qc.ca

Financement : Aucun reçu.

Remerciements : Les auteurs remercient Cynthia Tanguay, B. Sc., M. Sc., coordonnatrice, Unité de recherche en pratique pharmaceutique, $\mathrm{CHU}$ Sainte-Justine, pour l'aide à l'analyse des données et à la relecture du manuscrit.

\section{CJHP Subscriptions 2016 / Abonnements au JCPH 2016}

In 2015, we moved to an online only version of $C J H P$ and introduced the institutional online subscription option. CJHP online is included as a benefit of CSHP membership. All prices are in Canadian funds.

En 2015, nous avons migré vers la publication d'une seule version du $J C P H$, soit la copie électronique en ligne, et nous avons introduit en option un abonnement institutionnel. L'abonnement à la version électronique du $J C P H$ publiée en ligne est inclus dans les droits d'inscription à la SCPH. Tous les prix sont en dollars canadiens.

\begin{tabular}{|c|c|c|}
\hline $\begin{array}{l}\text { Subscriber group / } \\
\text { Groupe d'abonnés }\end{array}$ & $\begin{array}{l}\text { Individual Online Subscription / } \\
\text { Abonnement individuel en ligne }\end{array}$ & $\begin{array}{l}\text { Institutional Online Subscriptions / } \\
\text { Abonnement institutionnel en ligne }\end{array}$ \\
\hline $\begin{array}{l}\text { Nonmembers / } \\
\text { Non-membres }\end{array}$ & $\begin{array}{l}\$ 160.00 \text { per year, plus GST or HST } \\
160,00 \$ \text { par an, plus TPS ou TVH }\end{array}$ & $\begin{array}{l}\$ 480.00 \text { per year, plus GST or HST } \\
480,00 \$ \text { par an, plus TPS ou TVH }\end{array}$ \\
\hline
\end{tabular}

If you would like to purchase a subscription, please fill-out our CJHP 2016 Subscription Application Form, which can be found on the CJHP website: www.cjhp-online.ca. Please direct any comments or questions to cjhpedit@cshp.ca.

Si vous désirez vous abonner, veuillez remplir le formulaire d'abonnement au JCPH 2016. Vous pouvez l'obtenir en visitant le site Web du JCPH : www.cjhp-online.ca. Pour tout commentaire ou toute question, veuillez vous adresser à cjhpedit@cshp.ca. 